\section{Differential diagnosis between intraductal papillary mucinous neoplasm with an associated invasive carcinoma and pancreatic ductal adenocarcinoma on ultrasonography: the utility of echo intensity and contrast enhancement}

\author{
Masato Saito ${ }^{1}$, Naoki Hirokawa², Yoko Usami², Masanori Someya ${ }^{2}$, Koh-ichi Sakata ${ }^{2}$ \\ 'Department of Radiology, Sapporo Teishinkai Hospital, Sapporo; 'Department of Radiology, \\ Sapporo Medical University School of Medicine, Sapporo, Japan
}

Purpose: The aim of this study was to investigate the utility of echo intensity and contrast enhancement in the differential diagnosis between intraductal papillary mucinous neoplasm with an associated invasive carcinoma (IPMN-IC) and pancreatic ductal adenocarcinoma (PDAC) on ultrasonography.

Methods: This study included eight and 37 patients who had pathologically confirmed IPMN-IC and PDAC, respectively, and were enrolled for a comparative analysis of the sonographic features of the tumors. In the quantitative echo intensity evaluation, the two groups were compared with respect to the difference between the tumor intensity and the pancreatic intensity (TI-PI) and between the tumor intensity and the vascular intensity (TI-VI). In the quantitative contrast enhancement evaluation, the increase in echo intensity $(\Delta \mathrm{TI})$ and increase in echo intensity per unit of time (slope) were compared between the groups. The echo intensity and contrast enhancement were also compared between the two groups in patients with T3-T4 disease. In addition, the correlations of the histological type, tumor size, stromal type, and T factor with echogenicity and contrast enhancement were analyzed.

Results: IPMN-IC had significantly greater echo intensity and contrast enhancement than PDAC (TI-PI, $\mathrm{P}=0.004$; TI-VI, $\mathrm{P}=0.001 ; \Delta \mathrm{TI}, \mathrm{P}=0.012$; slope, $\mathrm{P}=0.002$ ). In T3-T4 disease, IPMN-IC also showed greater echo intensity and faster enhancement than PDAC. Echo intensity and contrast enhancement were correlated with histological type (TI-PI, $\mathrm{P}=0.003 ; \mathrm{TI}-\mathrm{VI}, \mathrm{P}<0.001 ; \Delta \mathrm{TI}$, $\mathrm{P}=0.007$; slope, $\mathrm{P}<0.001$ ).

Conclusion: IPMN-IC and PDAC can be differentiated by the quantitative evaluation of echo intensity and contrast enhancement.

Keywords: Ultrasonography; Sonazoid; Carcinoma, pancreatic ductal; Diagnosis, differential

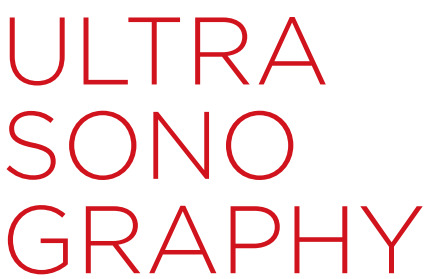

ORIGINAL ARTICLE

https://doi.org/10.14366/usg. 16039 pISSN: 2288-5919 - elSSN: 2288-5943 Ultrasonography 2017;36:260-269

Received: October 3, 2016

Revised: February 3, 2017

Accepted: February 4, 2017

Correspondence to:

Masato Saito, MD, PhD, Department of Radiology, Sapporo Teishinkai Hospital, N1.E.16, Higashi-ku, Sapporo 060-

0033, Japan

Tel. +81-11-712-1131

Fax. +81-11-751-0239

E-mail:ma_saito@teishinkai.jp

This is an Open Access article distributed under the terms of the Creative Commons Attribution NonCommercial License (http://creativecommons.org/ licenses/by-nc/3.0/) which permits unrestricted noncommercial use, distribution, and reproduction in any medium, provided the original work is properly cited.

Copyright @ 2017 Korean Society of Ultrasound in Medicine (KSUM)

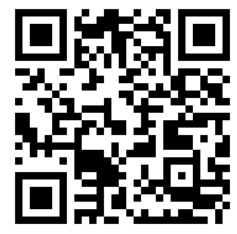

How to cite this article:

Saito M, Hirokawa N, Usami Y, Someya M Sakata K. Differential diagnosis between intraductal papillary mucinous neoplasm with an associated invasive carcinoma and pancreatic ductal adenocarcinoma on ultrasonography: the utility of echo intensity and contrast enhancement. Ultrasonography. 2017 Jul;36(3):260-269. 


\section{Introduction}

Pancreatic carcinoma ranks fourth among the causes of cancerrelated mortality in the United States and Europe, and it is predicted to be second to lung cancer by $2020[1,2]$. In addition, advanced cases of pancreatic carcinoma are common because early detection is difficult, and therefore the prognosis is poor, with a 5-year survival rate of less than $10 \%$ [3]. Although it is invasive, intraductal papillary mucinous carcinoma (IPMC) has been reported to have a better prognosis than pancreatic ductal adenocarcinoma (PDAC) [4-8], and therefore it is important to differentiate between the two types of cancer when developing a treatment strategy and estimating the prognosis. IPMC is the result of a malignant transformation from an intraductal papillary mucinous neoplasm (IPMN), but PDAC can coexist with an IPMN, and so it is necessary to differentiate between them [9-11].

No studies have been published evaluating the tumor echo intensity and contrast enhancement of IPMC and PDAC by transabdominal ultrasonography. In this study, we qualitatively and quantitatively compared the tumor echo intensity and contrast enhancement of IPMC and PDAC. We then investigated the usefulness of tumor echo intensity and contrast enhancement in the differential diagnosis between the two types of tumors, and ascertained the determining histopathological factors.

\section{Materials and Methods}

\section{Subjects}

This was a retrospective study approved by the Institutional Review Board of our hospital. The subjects were 57 patients who consecutively underwent ultrasonography for pancreatic neoplasms that were surgically resected and pathologically confirmed to be IPMC or PDAC between April 2007 and March 2012. Based on the 2010 World Health Organization classification, IPMC was subdivided into IPMN with high grade dysplasia (HD) and IPMN with an associated invasive carcinoma (IC). In order to ensure the reliability of the quantitative data, cases of IPMN with $H D(n=5)$ and minimally invasive IPMC $(n=2)$ were excluded from the study. Five other cases were excluded because of poor imaging of the neoplasm due to ultrasonic attenuation or interference by gastrointestinal gas. Forty-five cases were enrolled in the echo intensity evaluation. Of those 45 cases, 44 were included in the qualitative evaluation of contrast enhancement; one patient who had not undergone contrast-enhanced ultrasonography was excluded. For the quantitative analysis of contrast enhancement, three cases in which the contrast agent had already been injected in the initial frame or in which quantitative analysis was difficult due to poor respiratory

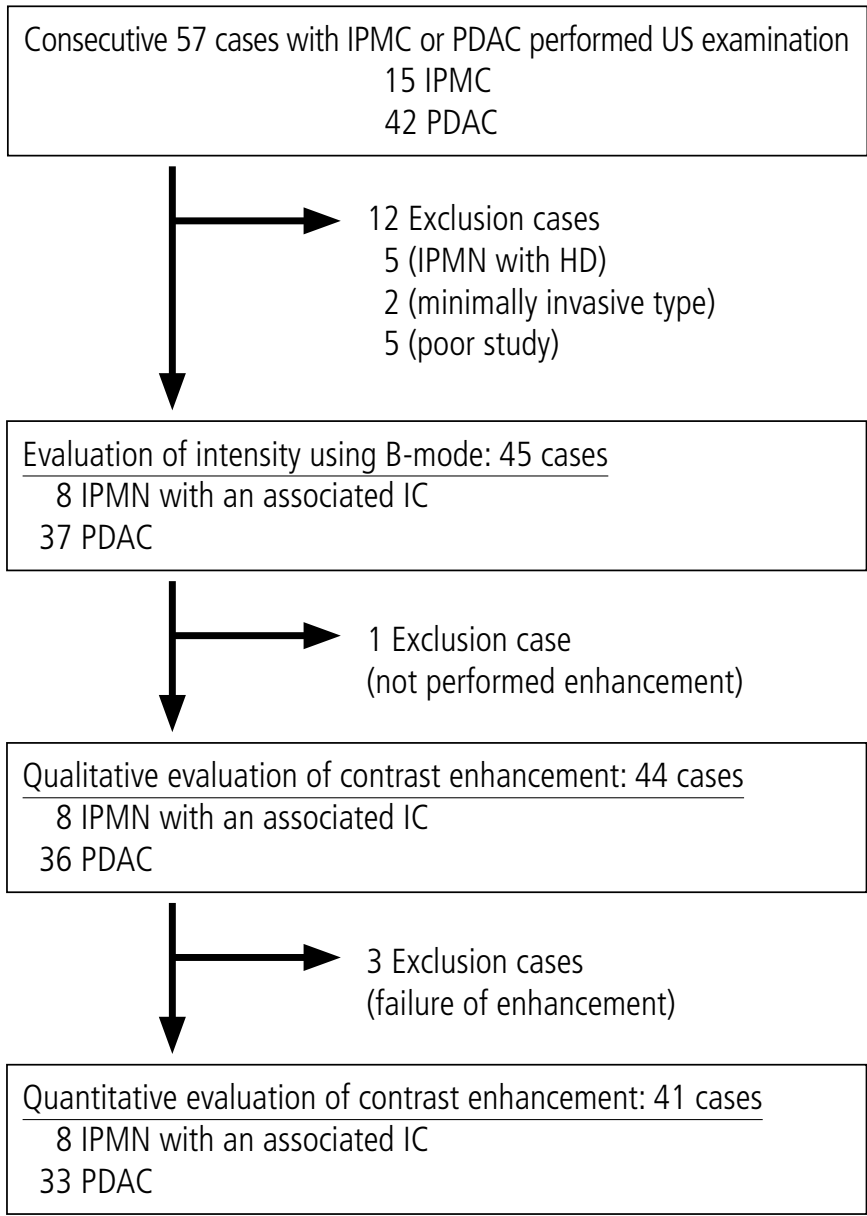

Fig. 1. Selection of the study population. IPMC, intraductal papillary mucinous carcinoma; PDAC, pancreatic ductal adenocarcinoma; US, ultrasonography; IPMN, intraductal papillary mucinous neoplasm; $H D$, high grade dysplasia; IC, invasive carcinoma.

control were excluded. Ultimately, 41 cases were included in the quantitative evaluation of contrast enhancement. The cases are summarized in Fig. 1.

\section{Scanning Procedures}

The ultrasound apparatuses used were the Aplio XV and Aplio XG (SSA770A, SSA790A; Toshiba Medical Systems Corp., Otawara, Japan), and the probes were $3.75-\mathrm{MHz}$ and $6-\mathrm{MHz}$ convex probes (PVT375BT, PVT674BT). The focal point was the bottom margin of the lesion. In the contrast-enhanced test, a uniform dose of $0.5 \mathrm{~mL}$ of the ultrasound contrast agent Sonazoid (Daiichi Sankyo, Tokyo, Japan) was administered in all cases. The imaging modes used were phase inversion and differential tissue harmonic imaging (D-THI). The acoustic power was fixed at $4 \%$ for the phase inversion mode (mechanical index $[\mathrm{MI}]=0.19-0.36$ ), and the Ml was fixed at 0.4 in the D-THI mode. Contrast enhancement was evaluated in the early 
vascular phase from 10 to 40 seconds.

\section{Image Analysis}

Ultrasonography was performed by a single radiologist with over 20 years of experience who had been certified by the Japan Society of Ultrasonics in Medicine. Another radiological specialist with over 10 years of experience performed the qualitative and quantitative analyses of intensity and contrast characteristics.

For the qualitative evaluation, tumor intensity was classified into the following four groups: (1) hypointense, (2) slightly hypointense, (3) isointense, and (4) hyperintense relative to the normal pancreatic parenchyma. Hypoechoic lesions with an intensity close to that of the vasculature that showed strong contrasts with the pancreatic parenchyma were defined as hypointense, while hypoechoic lesions with an intensity close to that of the pancreatic parenchyma were defined as slightly hypointense. They were scored as follows: 1 point, hypointense; 2 points, slightly hypointense; 3 points, isointense; and 4 points, hyperintense (Fig. 2).
For the quantitative analysis, the entire shape of the tumor was traced as the region of interest (ROI). For comparison, the normal pancreatic parenchyma and the primary vasculature in the vicinity were considered the ROI. The signal strength in the ROI was quantified using US Image Lab ver. 6.4 (Toshiba Medical Systems Corp., Tokyo, Japan). The quantitative data were used to establish two parameters: the difference between the tumor intensity and the pancreatic intensity (TI-PI), and the difference between the tumor intensity and the vascular intensity (TI-VI).

In the qualitative evaluation, contrast enhancement was classified into the following four categories: (1) not stained, (2) slightly/ marginally stained, (3) well stained but weaker than the pancreatic parenchyma, and (4) well stained and equivalent to the pancreatic parenchyma, as shown in Fig. 3. They were scored as follows: 1 point, not stained; 2 points, slightly/marginally stained; 3 points, well stained but weaker than the pancreatic parenchyma; and 4 points, well stained and equivalent to the pancreatic parenchyma.

In the quantitative evaluation, the point in time when the contrast

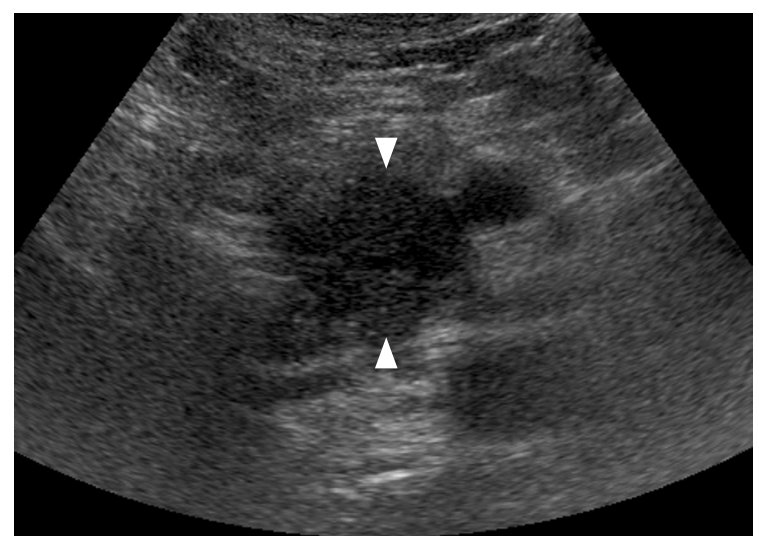

A

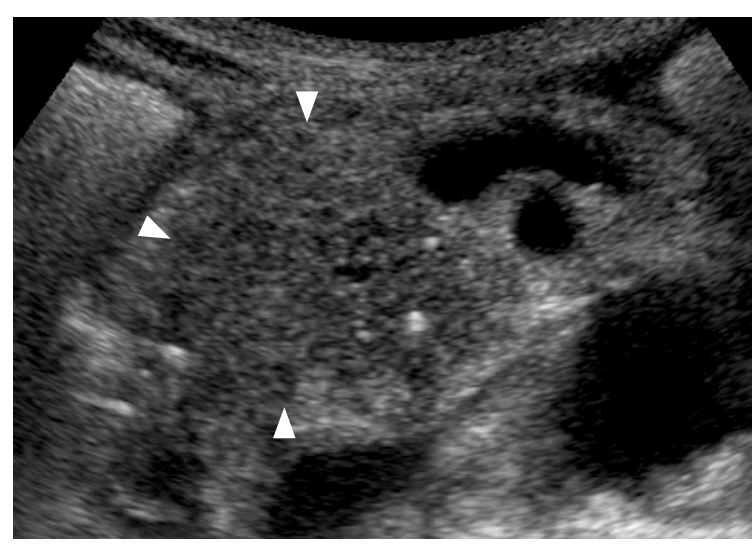

C

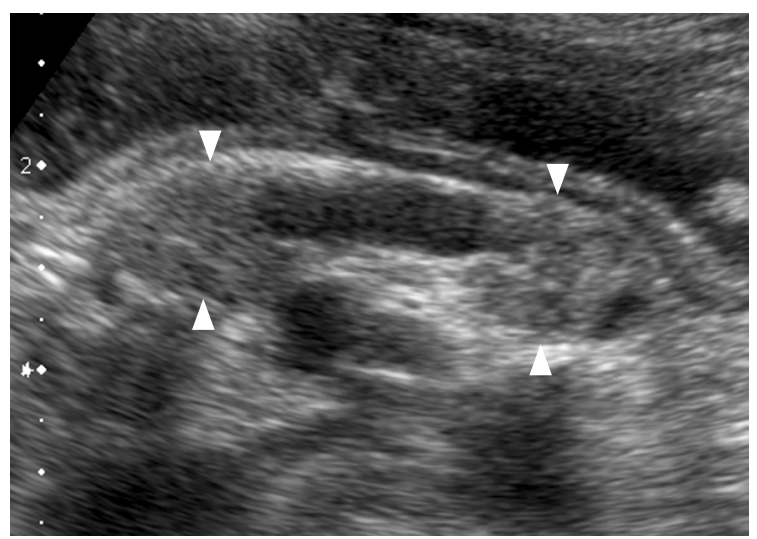

B

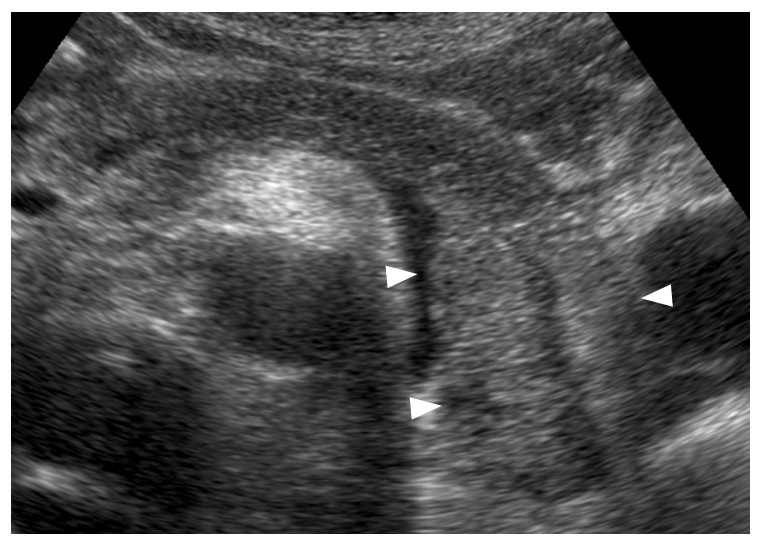

D

Fig. 2. Definition of echo intensity scores.

A-D. In the qualitative analysis, the echo intensity of the tumor (arrowheads) was categorized and scored as follows: (A) hypointense, 1 point; (B) slightly hypointense, 2 points; (C) isointense, 3 points; and (D) hyperintense, 4 points. 


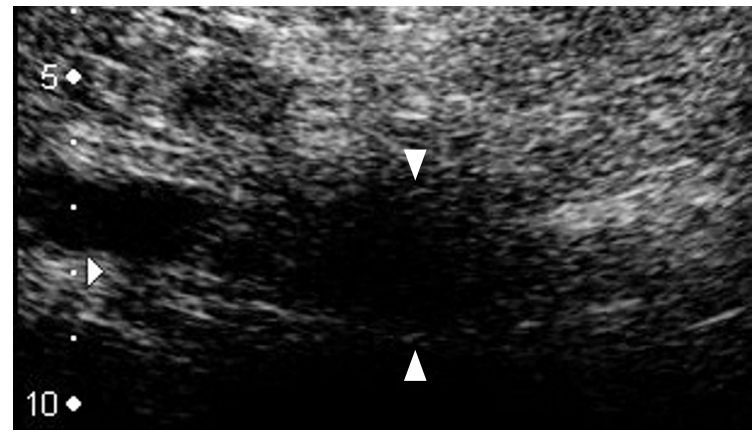

A

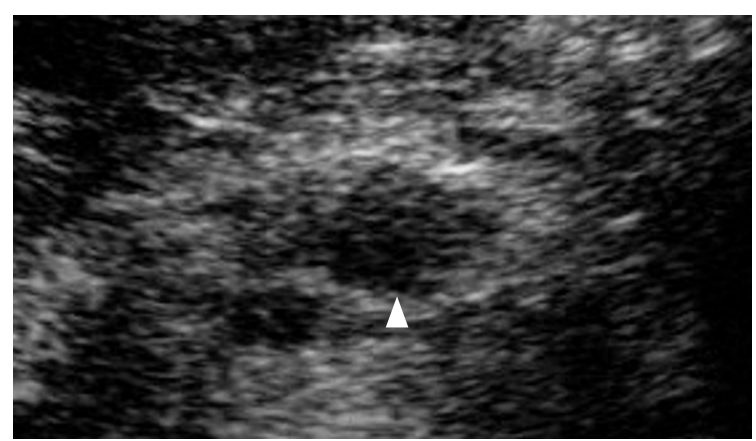

B

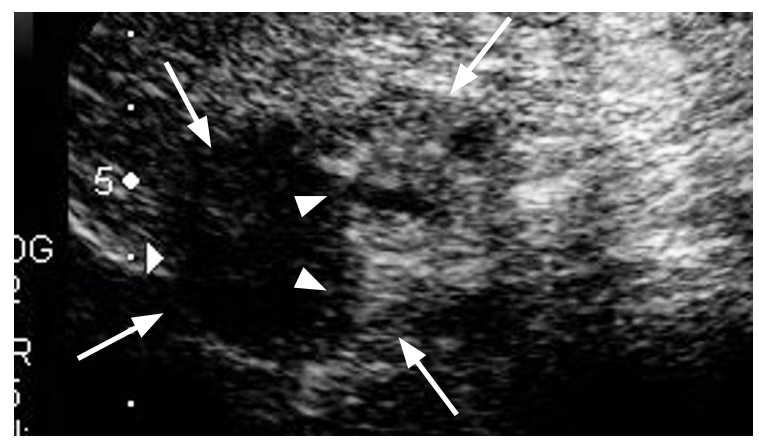

D

agent flowed into the abdominal aorta or the superior mesenteric artery in the vicinity of the tumor was set as the start of contrast enhancement. The chronological changes in the signal strength in the ROI set at the center of the solid part of the tumor were measured using Image Lab ver. 3.01 (Toshiba Medical Systems Corp., Tokyo, Japan). The ROI for IPMN-IC was placed in the center of the mural nodule in a way that enabled the exclusion of cystic lesions and branched pancreatic ducts from the ROI. The histopathology in the ROI was adenocarcinoma, not adenoma. The parameters of the increase in echo intensity $(\Delta \mathrm{TI})$ and echo intensity per unit of time (slope) were used to evaluate contrast enhancement.

\section{Evaluation of the Variables}

The echo intensity and contrast enhancement of both groups were

\section{Fig. 3. Definition of contrast scores.}

A-E. In the qualitative analysis, contrast enhancement of the tumor (arrowheads) was categorized and scored as follows: (A) not stained, 1 point; (B) slightly stained or (C) marginally stained, 2 points; (D) well stained but weaker than the pancreatic parenchyma, 3 points; and (E) well stained and equivalent to the pancreatic parenchyma, 4 points. In a tumor with mixed components (arrows in D), mural nodule enhancement (arrowheads) was clearly distinguished from the nonenhancing cystic component.

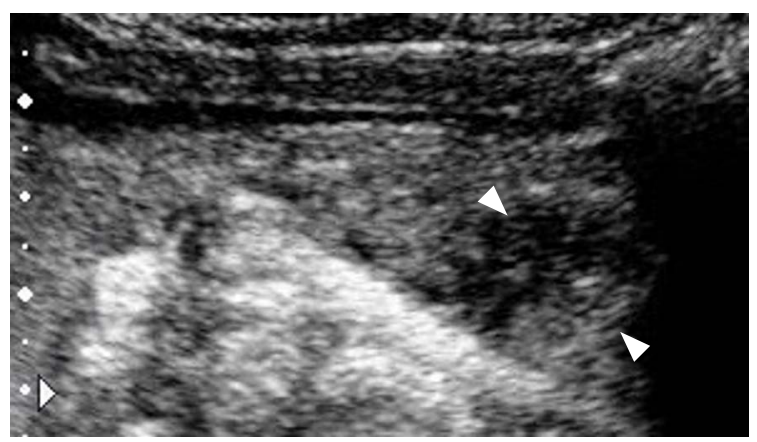

C

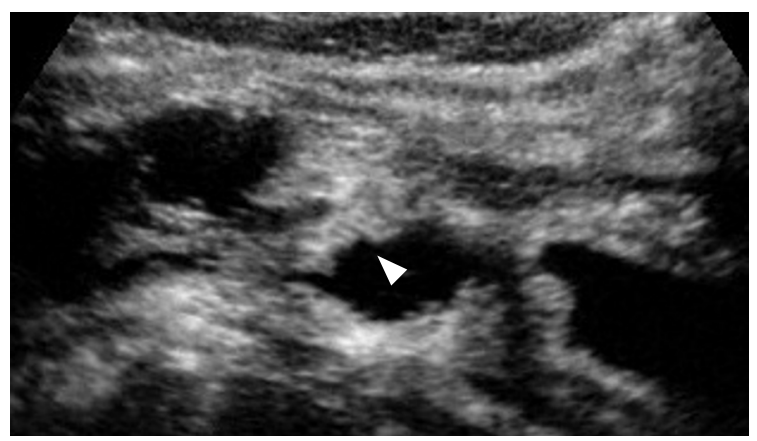

E

qualitatively and quantitatively compared to validate the usefulness of the ultrasonic differential diagnosis of IPMN-IC and PDAC. First, the qualitative echo intensity scores and contrast enhancement scores were compared between the two groups. TI-PI and TI-VI were used to compare the quantitative echo intensity between the two groups, and $\Delta \mathrm{TI}$ and slope were used to compare the quantitative contrast characteristics.

In addition, to compare patients at the same tumor stage, the echo intensity parameters (TI-PI and TI-VI) and the contrast enhancement parameters ( $\Delta \mathrm{TI}$ and slope) were compared between the two groups among patients with T3-T4 disease.

In order to verify the histopathological factors pertinent to echo intensity and contrast enhancement, multiple regression analysis was performed with the histopathological type, tumor size, stromal 
type, and $T$ factor as variables included in the analysis.

\section{Statistical Analysis}

The Mann-Whitney $\mathrm{U}$ test was used to compare the qualitative echo intensity scores and the qualitative contrast enhancement scores between the two groups. For the quantitative comparison of echo intensity and contrast enhancement, the $t$ test was used for the parametric data and the Mann-Whitney $U$ test was used for the nonparametric data. Multiple regression analysis was used for the factor analysis of echo intensity and contrast enhancement. SPSS ver. 22.0 (IBM Corp., Armonk, NY, USA) was used for the statistical analysis, and the statistical significance threshold was set at $\mathrm{P}<0.05$.

\section{Results}

\section{Subject Characteristics and Pathological Factors}

The characteristics of the subjects are shown in Table 1. The T factor was classified into two groups (T1-T2 and T3-T4) according to the seventh edition of the Union for International Cancer Control classification.

No significant differences were found between the IPMN-IC group and the PDAC group in terms of age, sex, body mass index, neoplasm location, tumor size, $\mathrm{T}$ factor, or stromal type.

\section{Qualitative Evaluation of Echo Intensity}

The IPMN-IC group included four hypointense cases (50.0\%), three slightly hypointense cases (37.5\%), and one hyperintense case $(12.5 \%)$, while the PDAC group included 32 hypointense cases
(86.5\%), four slightly hypointense cases (10.8\%), one isointense case $(2.7 \%)$, and no hyperintense cases. The average qualitative score was significantly higher in the IPMN-IC group (IPMN-IC, 1.75 $\pm 1.03 ;$ PDAC, 1.14 $\pm 0.42 ; P=0.009$ ).

\section{Quantitative Evaluation of Echo Intensity}

The box-and-whisker plots of the TI-PI and TI-VI results for the IPMN-IC and PDAC groups are shown in Fig. 4. IPMN-IC was found to have a significantly higher TI-PI difference than PDAC (IPMNIC, $-8.0 \pm 8.6 \mathrm{~dB} ; \mathrm{PDAC},-15.3 \pm 5.7 \mathrm{~dB} ; \mathrm{P}=0.004)$. A similar result was noted for TI-VI (IPMN-IC, $13.9 \pm 5.6 \mathrm{~dB}$; PDAC, $6.1 \pm 5.6 \mathrm{~dB}$; $\mathrm{P}=0.001)$.

The box-and-whisker plots of the TI-PI and TI-VI results for the IPMN-IC and PDAC in patients with T3-T4 disease are shown in Fig. 5. The intensity of IPMN-IC was significantly higher than that of PDAC (TI-PI, P=0.001; TI-VI, P<0.001).

\section{Qualitative Evaluation of Contrast Enhancement}

In the IPMN-IC group $(n=8)$, there were six cases that were well stained but weaker than the pancreatic parenchyma $(75 \%)$, and two cases that were well stained and equivalent to the pancreatic parenchyma (25.0\%). In the PDAC group, there were six cases that were not stained (16.7\%), 15 cases that were slightly/marginally stained $(41.7 \%), 13$ cases that were well stained but weaker than the pancreatic parenchyma (36.1\%), and two cases that were well stained and equivalent to the pancreatic parenchyma (5.5\%). IPMNIC had significantly higher qualitative scores than PDAC (IPMN-IC, 3.2 $\pm 0.4 ;$ PDAC, $2.3 \pm 0.8 ; P=0.003)$.

Table 1. Characteristics of the subjects

\begin{tabular}{|c|c|c|c|c|}
\hline & & IPMN-IC $(n=8)$ & PDAC $(n=37)$ & P-value \\
\hline \multirow[t]{2}{*}{ Gender } & Male & 3 & 19 & $0.699^{a)}$ \\
\hline & Female & 5 & 18 & \\
\hline $\mathrm{BMI}\left(\mathrm{kg} / \mathrm{m}^{2}\right)$ & & $20.16 \pm 2.43$ & $20.83 \pm 3.71$ & $>0.990^{c)}$ \\
\hline Location & $\mathrm{Ph}$ & 5 & 24 & $>0.990^{\mathrm{a})}$ \\
\hline \multirow[t]{2}{*}{ Stromal type } & Scirrhous & 1 & 12 & $0.405^{\mathrm{a})}$ \\
\hline & Other & 7 & 25 & \\
\hline \multirow[t]{2}{*}{ Tfactor } & $\mathrm{T} 1-\mathrm{T} 2$ & 2 & 6 & $0.618^{a)}$ \\
\hline & $\mathrm{T} 3-\mathrm{T} 4$ & 6 & 31 & \\
\hline
\end{tabular}

Values are presented as number or mean \pm SD.

IPMN-IC, intraductal papillary mucinous neoplasm with an associated invasive carcinoma; PDAC, pancreatic ductal adenocarcinoma; BMI, body mass index; Ph, pancreatic head; Pb, pancreatic body; Pt, pancreatic tail; SD, standard deviation.

${ }^{\text {a) }}$ Fisher test. ${ }^{\text {b) }}$ Student t-test. ${ }^{\text {c } M a n n-W h i t n e y ~ U ~ t e s t . ~}$ 


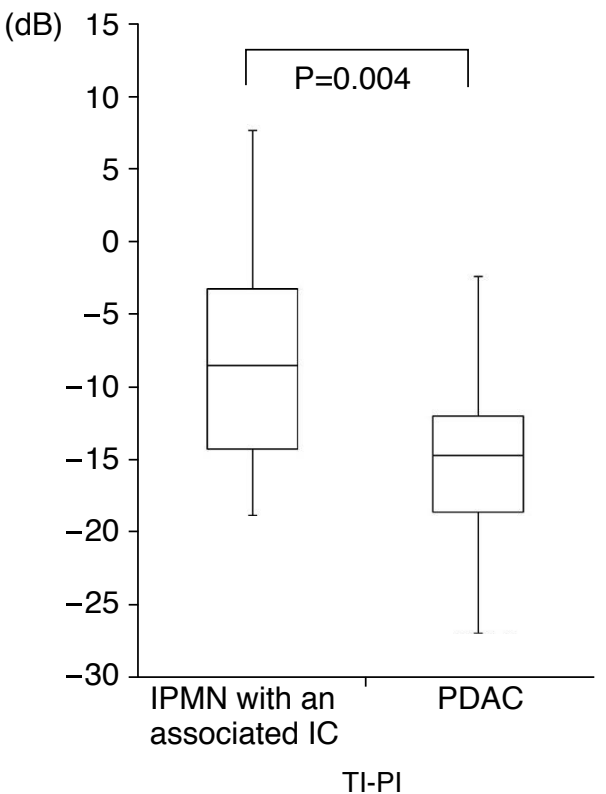

A

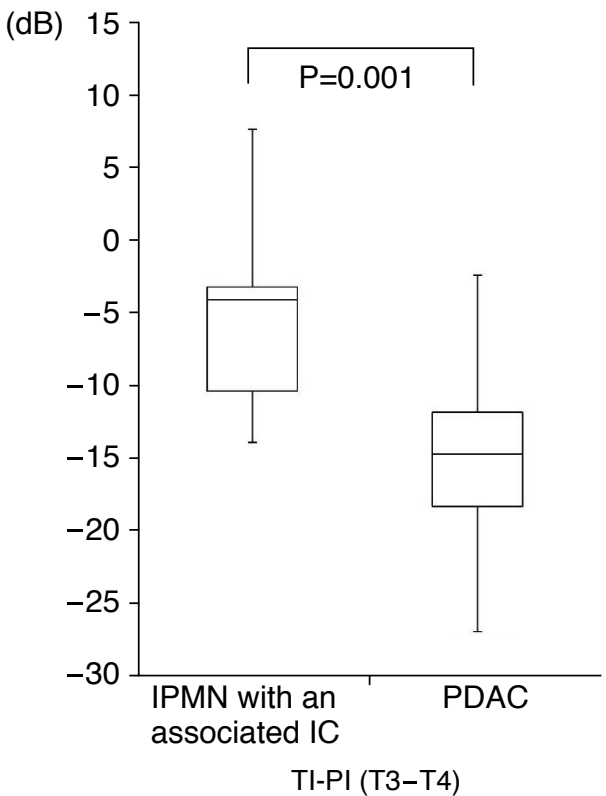

A

Quantitative Evaluation of Contrast Enhancement The box-and-whisker plots of $\triangle \mathrm{TI}$ and slope for the IPMN-IC and PDAC groups are shown in Fig. 6. IPMN-IC exhibited a significantly greater $\triangle \mathrm{TI}$ than PDAC (IPMN-IC, $14.9 \pm 4.4 \mathrm{~dB}$; PDAC, $8.8 \pm 6.0 \mathrm{~dB}$; $P=0.012$ ). IPMN-IC also exhibited a significantly greater slope than PDAC (IPMN-IC, $1.8 \pm 0.6 \mathrm{~dB} / \mathrm{sec} ;$ PDAC, $0.8 \pm 0.6 \mathrm{~dB} / \mathrm{sec} ; \mathrm{P}=0.002$ ).

The contrast enhancement parameters of $\triangle \mathrm{TI}$ and slope were compared between the two groups of patients with T3-T4 disease.

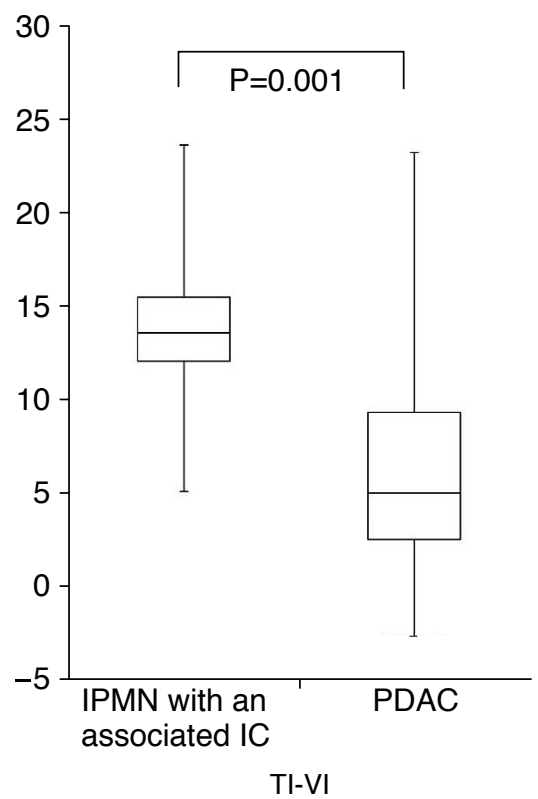

Fig. 4. Comparison of the echo intensities between intraductal papillary mucinous neoplasm (IPMN) with an associated invasive carcinoma (IC) and pancreatic ductal adenocarcinoma (PDAC).

$A$, B. The echo intensities of IPMN with an associated IC were higher than those observed for PDAC. $\mathrm{TI}$, tumor intensity; PI, pancreatic intensity; VI, vascular intensity.
(dB)

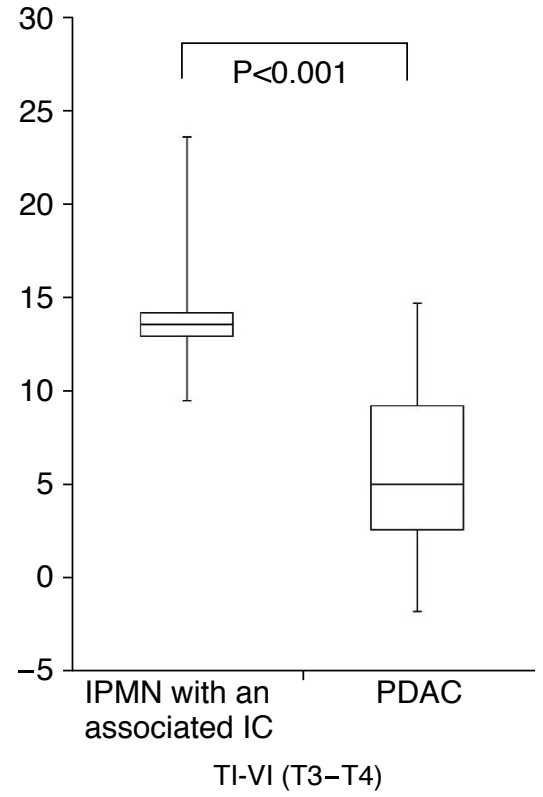

Fig. 5. Comparison of the echo intensities of intraductal papillary mucinous neoplasm (IPMN) with an associated invasive carcinoma (IC) and pancreatic ductal adenocarcinoma (PDAC) in patients with T3-T4 disease.

A, B. In T3-T4 disease, the echo intensity of IPMN with an associated IC was higher than that of PDAC. TI, tumor intensity; PI, pancreatic intensity; $\mathrm{VI}$, vascular intensity. 
(dB)

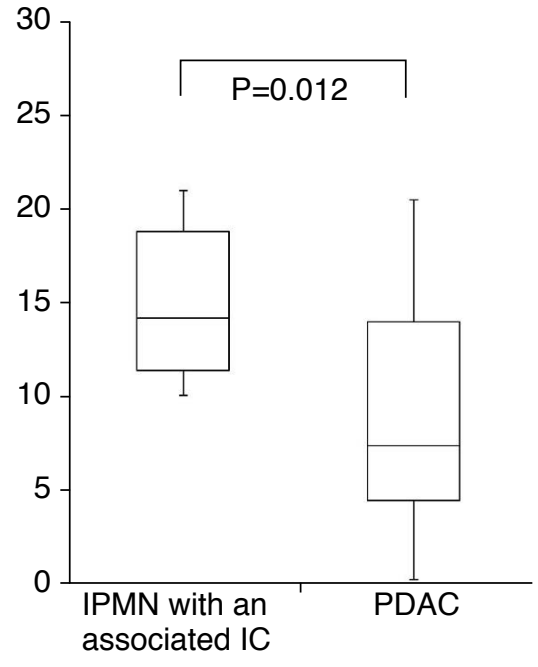

$\triangle \mathrm{TI}$
(dB/sec)

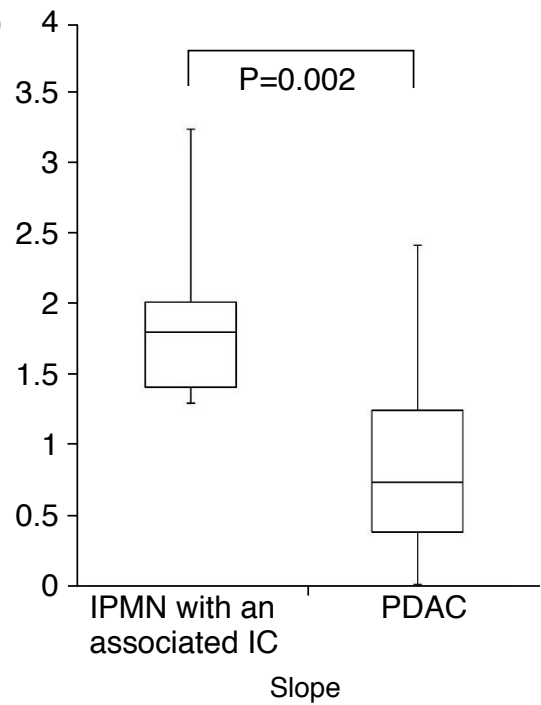

Fig. 6. Comparison of tumor enhancement between intraductal papillary mucinous neoplasm (IPMN) with an associated invasive carcinoma (IC) and pancreatic ductal adenocarcinoma (PDAC).

$A, B$. Contrast enhancement of IPMN with an associated IC was greater than that of PDAC. $\triangle \mathrm{TI}$, the increase in echo intensity; slope, the increase in echo intensity per unit time.

B

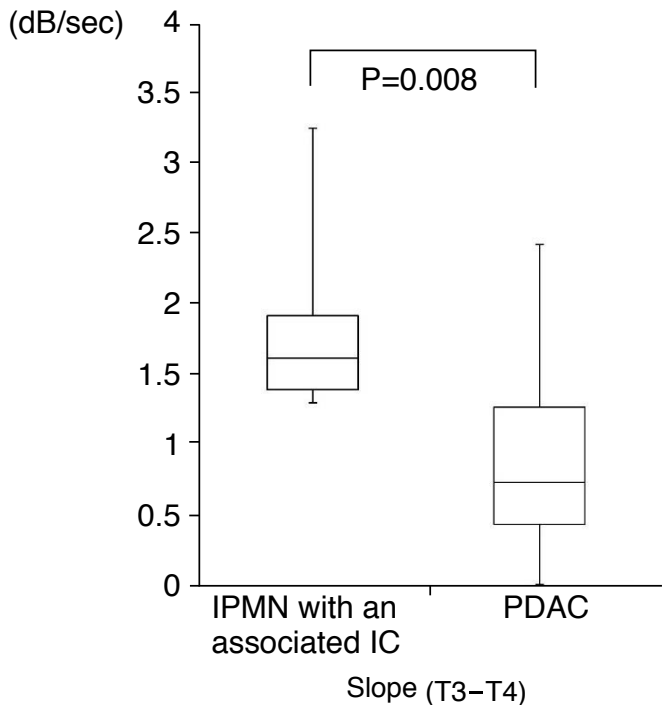

Fig. 7. Comparison of contrast enhancement between intraductal papillary mucinous neoplasm (IPMN) with an associated invasive carcinoma (IC) and pancreatic ductal adenocarcinoma (PDAC) in patients with T3-T4 disease.

A. In T3-T4 disease, a significant difference in the increase in echo intensity $(\Delta T I)$ was not seen between the two groups. B. In T3-T4 disease, the slope of IPMN with an associated IC was greater than that of PDAC. Slope, the increase in echo intensity per unit time.

factors with tumor echo intensity and contrast enhancement are shown in Tables 2 and 3.

Histological type was found to be correlated with all intensity and contrast parameters (TI-PI, $\mathrm{P}=0.003$; $\mathrm{TI}-\mathrm{VI}, \mathrm{P}<0.001 ; \Delta \mathrm{TI}, \mathrm{P}=0.007$; slope, $P<0.001)$. In contrast, no significant correlations were observed among the tumor size, stromal type, or $\mathrm{T}$ factor with any of the intensity parameters or contrast enhancement parameters.

\section{Discussion}

In the diagnosis of pancreatic carcinoma, transabdominal ultrasonography is used not only for detecting small lesions but also for qualitative diagnoses and assessing disease progression because it is simple, noninvasive, and has superior resolution. In recent years, contrastenhanced transabdominal ultrasonography has been applied to the pancreas as well, improving the diagnostic potential of qualitative evaluations [12-15]. It enables observation of the amount of blood flow to the neoplasm, the detection of small lesions, and the acquisition of useful information such as the extent of vascular invasion. Studies of contrast-enhanced endoscopic ultrasonography, which has high spatial resolution, have also occasionally been published [16-22]

Pancreatic carcinoma is an illness with difficulties in early detection and a poor prognosis, but among pancreatic carcinomas, 
Table 2. Results of the multiple regression analysis of the intensity parameters

\begin{tabular}{|c|c|c|c|c|c|c|c|c|}
\hline & \multicolumn{4}{|c|}{ TI-PI } & \multicolumn{4}{|c|}{ TI-VI } \\
\hline & \multirow{2}{*}{$\beta$} & \multirow{2}{*}{ P-value } & \multicolumn{2}{|c|}{$95 \% \mathrm{Cl}$} & \multirow{2}{*}{$\beta$} & \multirow{2}{*}{ P-value } & \multicolumn{2}{|c|}{$95 \% \mathrm{Cl}$} \\
\hline & & & Lower & Upper & & & Lower & Upper \\
\hline Histological type & -0.461 & 0.003 & -13.360 & -2.948 & -0.534 & $<0.001$ & -13.407 & -4.132 \\
\hline Size & -0.183 & 0.238 & -0.246 & 0.063 & -0.257 & 0.087 & -0.257 & 0.018 \\
\hline Stromal type & 0.001 & 0.999 & -4.375 & 4.379 & 0.063 & 0.651 & -3.020 & 4.778 \\
\hline T factor & 0.207 & 0.179 & -1.752 & 9.066 & 0.102 & 0.487 & -3.147 & 6.489 \\
\hline
\end{tabular}

$\mathrm{TI}$, tumor intensity; PI, pancreatic intensity; $\mathrm{VI}$, vascular intensity; $\beta$, standardized partial regression coefficient; $\mathrm{Cl}$, confidence interval.

Table 3. Results of the multiple regression analysis of the contrast parameters

\begin{tabular}{|c|c|c|c|c|c|c|c|c|}
\hline & \multicolumn{4}{|c|}{$\Delta \mathrm{TI}$} & \multicolumn{4}{|c|}{ Slope } \\
\hline & \multirow{2}{*}{$\beta$} & \multirow{2}{*}{ P-value } & \multicolumn{2}{|c|}{$95 \% \mathrm{Cl}$} & \multirow{2}{*}{$\beta$} & \multirow{2}{*}{ P-value } & \multicolumn{2}{|c|}{$95 \% \mathrm{Cl}$} \\
\hline & & & Lower & Upper & & & Lower & Upper \\
\hline Histological type & -0.445 & 0.007 & -11.873 & -1.976 & -0.577 & $<0.001$ & -1.634 & -0.540 \\
\hline Size & -0.148 & 0.387 & -0.217 & 0.086 & -0.097 & 0.533 & -0.022 & 0.012 \\
\hline Stromal type & 0.173 & 0.275 & -1.995 & 6.808 & 0.231 & 0.114 & -0.098 & 0.875 \\
\hline T factor & 0.096 & 0.567 & -3.931 & 7.063 & 0.062 & 0.685 & -0.485 & 0.730 \\
\hline
\end{tabular}

$\Delta \mathrm{TI}$, the increase in echo intensity; slope, the increase in echo intensity per unit time; $\beta$, standardized partial regression coefficient; $\mathrm{Cl}$, confidence interval.

IPMC has a better prognosis than PDAC. The 5-year survival rate of invasive IPMN has been reported to be $34 \%-43 \%$, while that of PDAC is $9 \%-21 \%[4-8,11]$. In addition, IPMC was further subdivided into carcinoma in situ, minimally IC, and IC in the Japan Pancreatic Cancer Registry Report 2007, with 5-year survival rates of $84.2 \%, 73.2 \%$, and $37.6 \%$ respectively, compared to $7.9 \%$ for PDAC [3]. Thus, a difference in prognosis can be seen depending on the histological type and degree of invasiveness. Some authors have suggested that this is because IPMC has relatively quiescent biological characteristics compared to PDAC, and is discovered at an earlier stage [11]. Thus, differentiating between PDAC and IPMC, particularly IPMN-IC (IC arising in IPMN), is important for estimating a patient's prognosis and developing a treatment strategy, but no studies have yet explored the use of imaging for differentiation between these types of cancer. We focused on tumor echo intensity and contrast enhancement in our investigation of the possibility of performing a differential diagnosis between IPMNIC and PDAC using transabdominal ultrasonography. In addition, we histopathologically verified the factors that determined tumor intensity and contrast enhancement.

In this study, we found that IPMN-IC had a qualitatively and quantitatively higher intensity than PDAC. In addition, echo intensity was also found to differ according to histological type in patients with T3-T4 disease. No prior studies have been published of histological differentiation based on differences in echogenicity. Our results constitute the first report regarding tumor intensity in cases of IPMN-IC (IC arising from IPMN), with the characteristic finding that IPMN-IC was found to have a higher intensity than PDAC. As for the reason why IPMN-IC has a higher intensity than PDAC, we surmise that the morphological characteristics of neoplasms that form papillary projections in the cystic lumen manifest as differences in echo intensity. In other words, it is conceivably possible that the numerous minute gaps among papillary neoplasms cause scattering and multiple reflections, and furthermore that there are histological heterogeneities resulting from mucus interposed in the spaces formed by the minute gaps, increasing the intensity. The histopathology of the high-intensity PDAC showed cancerous ducts aggregated in the tumor, and the minute gaps inside the ducts are believed to have contributed to the high intensity.

No prior studies have been conducted on the echo intensity of IPMN-IC (IC arising in IPMN). The authors of a paper reporting the sonographic findings associated with invasive micropapillary carcinoma of the breast discussed the correlation between the echo intensity and histopathological nature of neoplasms, and stated that tumors that did not exhibit an enlarged central lumen in tumor cell clusters had a low echo intensity, whereas cases exhibiting an enlarged central lumen had a relatively high intensity [23]. This also indicates that the presence of minute gap spaces is correlated with echo intensity, supporting our proposed rationale for the high IPMNIC intensity. 
In this study, IPMN-IC exhibited quantitatively and qualitatively greater contrast enhancement than PDAC. Moreover, in the comparison between patients with T3-T4 disease in the two groups, the slope of IPMN-IC was greater than that of PDAC. Furthermore, histological type itself was shown to be a factor determining contrast enhancement. Takeshima et al. [12] showed that papillary carcinoma was well stained relative to the pancreatic parenchyma, whereas tubular adenocarcinoma was weakly stained with contrastenhanced ultrasonography. In other words, this fact indicated that the histological type of a pancreatic carcinoma had an effect on ultrasonic contrast enhancement. As differences in histological type were reflected in contrast enhancement in this study, we surmise the following. Because the ultrasound contrast agent does not seep into the stroma [24], high vascular density in the tumor and large average blood vessel size are factors underlying good contrast enhancement $[25,26]$. The present results suggest that IPMN-IC contains more tumor vasculature and has a lower vascular resistance than PDAC. It is conceivable that in lesions with papillary projections that proliferate and protrude into a lumen, as is the case for IPMN, tumor vasculature could be induced, increasing tumor growth and increasing contrast penetration. Differences in peripheral vascular resistance, which are indicative of differences in vascular size, are reflected in slope, and so it is conceivable that PDAC more easily causes vascular stenosis and occlusion as a result of that invasion, increasing vascular resistance.

In terms of the diagnostic modality, it is difficult to evaluate continuous contrast enhancement in a tumor using computed tomography or magnetic resonance imaging. In contrast, ultrasonography can be used to evaluate contrast enhancement accurately and continuously because it offers superior temporal resolution. Ultrasonography also has superior spatial resolution and is known to be useful in detecting microcystic lesions in a tumor as well as small mural nodules. Furthermore, this study showed that echo intensity was useful in differentiating histological types because it reflected the tissue structure of the tumor, thereby suggesting the superiority of ultrasonography in this context.

A limitation of the present study is the problem of the universality of lesion representation by transabdominal ultrasonography. The procedure is highly dependent on patient factors and the operator, and there are cases that are difficult to evaluate due to a deep lesion or gastrointestinal gas. However, we minimized this problem in the present study by analyzing results obtained by a single operator who is an ultrasonography specialist with over 20 years of experience and who specializes in pancreatic disorders.

A second limitation is the small sample size. It is necessary to accumulate more cases. A third limitation is that as a retrospective study, the reliability of the evaluations, including the setting of ROIs, is not guaranteed. However, the results from the qualitative and qualitative evaluations were similar, suggesting that the ROI settings achieved a certain standard of rigor.

The current study shows that a diagnosis of the histological type of pancreatic lesions is possible through an evaluation of echo intensity and contrast enhancement, in addition to the superior spatial and temporal resolution of transabdominal ultrasonography, which was useful in differentiating between IPMN-IC and PDAC. In particular, the qualitative evaluation was supported quantitatively, enabling differentiation to be made at the time and place of ultrasonography in clinical cases.

ORCID: Masato Saito: http://orcid.org/0000-0002-2567-6272; Naoki Hirokawa: http://orcid.org/0000-0002-7346-889X; Yoko Usami: http://orcid.org/0000-00032230-5457; Masanori Someya: http://orcid.org/0000-0001-8821-7754; Koh-ichi Sakata: http://orcid.org/0000-0002-9900-9632

\section{Conflict of Interest}

No potential conflict of interest relevant to this article was reported.

\section{Acknowledgments}

The authors thank Naohisa Kamiyama, Global Manager of Clinical Technology, Ultrasound General Imaging, GE Healthcare, for providing advice regarding the ImageLab software, and Masanori Nojima, instructor in the Department of Public Health, Sapporo Medical University, for guidance in performing the statistical analyses.

\section{References}

1. Rahib L, Smith BD, Aizenberg R, Rosenzweig AB, Fleshman JM, Matrisian LM. Projecting cancer incidence and deaths to 2030: the unexpected burden of thyroid, liver, and pancreas cancers in the United States. Cancer Res 2014;74:2913-2921.

2. Malvezzi M, Bertuccio P, Rosso T, Rota M, Levi F, La Vecchia C, et al. European cancer mortality predictions for the year 2015: does lung cancer have the highest death rate in EU women? Ann Oncol 2015;26:779-786.

3. Egawa S, Toma H, Ohigashi H, Okusaka T, Nakao A, Hatori T, et al. A digest of the Pancreatic Cancer Registry Report 2007. J Jpn Pancreas Soc 2008;23:105-123.

4. Woo SM, Ryu JK, Lee SH, Yoo JW, Park JK, Kim YT, et al. Survival and prognosis of invasive intraductal papillary mucinous neoplasms of the pancreas: comparison with pancreatic ductal adenocarcinoma. Pancreas 2008;36:50-55.

5. Murakami Y, Uemura K, Sudo T, Hayashidani Y, Hashimoto Y, Nakashima A, et al. Invasive intraductal papillary-mucinous 
neoplasm of the pancreas: comparison with pancreatic ductal adenocarcinoma. J Surg Oncol 2009;100:13-18.

6. Maire F, Hammel P, Terris B, Paye F, Scoazec JY, Cellier C, et al. Prognosis of malignant intraductal papillary mucinous tumours of the pancreas after surgical resection: comparison with pancreatic ductal adenocarcinoma. Gut 2002;51:717-722.

7. Shimada K, Sakamoto Y, Sano T, Kosuge T, Hiraoka N. Invasive carcinoma originating in an intraductal papillary mucinous neoplasm of the pancreas: a clinicopathologic comparison with a common type of invasive ductal carcinoma. Pancreas 2006;32:281287.

8. Sohn TA, Yeo CJ, Cameron JL, Hruban RH, Fukushima N, Campbell $K A$, et al. Intraductal papillary mucinous neoplasms of the pancreas: an updated experience. Ann Surg 2004;239:788-797.

9. Uehara H, Nakaizumi A, Ishikawa O, lishi H, Tatsumi K, Takakura $R$, et al. Development of ductal carcinoma of the pancreas during follow-up of branch duct intraductal papillary mucinous neoplasm of the pancreas. Gut 2008;57:1561-1565.

10. Tada M, Kawabe T, Arizumi M, Togawa O, Matsubara S, Yamamoto $\mathrm{N}$, et al. Pancreatic cancer in patients with pancreatic cystic lesions: a prospective study in 197 patients. Clin Gastroenterol Hepatol 2006;4:1265-1270.

11. Yamaguchi K, Kanemitsu S, Hatori T, Maguchi H, Shimizu Y, Tada $M$, et al. Pancreatic ductal adenocarcinoma derived from IPMN and pancreatic ductal adenocarcinoma concomitant with IPMN. Pancreas 2011;40:571-580.

12. Takeshima K, Kumada T, Toyoda H, Kiriyama S, Tanikawa M, Ichikawa $\mathrm{H}$, et al. Comparison of IV contrast-enhanced sonography and histopathology of pancreatic cancer. AJR Am J Roentgenol 2005; 185:1193-1200.

13. Ozawa Y, Numata K, Tanaka K, Ueno N, Kiba T, Hara K, et al. Contrast-enhanced sonography of small pancreatic mass lesions. J Ultrasound Med 2002;21:983-991.

14. Sofuni A, lijima H, Moriyasu F, Nakayama D, Shimizu M, Nakamura K, et al. Differential diagnosis of pancreatic tumors using ultrasound contrast imaging. J Gastroenterol 2005;40:518-525.

15. Pezzilli R, Serra C, Calculli L, Ferroni F, Iammarino MT, Casadei $R$. Three-dimensional contrast-enhanced ultrasonography of intraductal papillary mucinous neoplasms of the pancreas: a comparison with magnetic resonance imaging. Pancreas 2013;42:1164-1168.

16. Harima H, Kaino S, Shinoda S, Kawano M, Suenaga S, Sakaida I. Differential diagnosis of benign and malignant branch duct intraductal papillary mucinous neoplasm using contrast-enhanced endoscopic ultrasonography. World J Gastroenterol 2015;21:62526260.

17. Park JS, Kim HK, Bang BW, Kim SG, Jeong S, Lee DH. Effectiveness of contrast-enhanced harmonic endoscopic ultrasound for the evaluation of solid pancreatic masses. World J Gastroenterol 2014;20:518-524.

18. Kitano M, Kudo M, Yamao K, Takagi T, Sakamoto H, Komaki T, et al. Characterization of small solid tumors in the pancreas: the value of contrast-enhanced harmonic endoscopic ultrasonography. Am J Gastroenterol 2012;107:303-310.

19. Kitano M, Kudo M, Maekawa K, Suetomi Y, Sakamoto H, Fukuta $\mathrm{N}$, et al. Dynamic imaging of pancreatic diseases by contrast enhanced coded phase inversion harmonic ultrasonography. Gut 2004;53:854-859.

20. Sakamoto H, Kitano M, Suetomi Y, Maekawa K, Takeyama Y, Kudo M. Utility of contrast-enhanced endoscopic ultrasonography for diagnosis of small pancreatic carcinomas. Ultrasound Med Biol 2008;34:525-532.

21. Sakamoto H, Kitano M, Komaki T, Imai H, Kamata K, Kimura M, et al. Small invasive ductal carcinoma of the pancreas distinct from branch duct intraductal papillary mucinous neoplasm. World J Gastroenterol 2009;15:5489-5492.

22. Ohno E, Itoh A, Kawashima $H$, Ishikawa $T$, Matsubara $H$, Itoh $Y$, et al. Malignant transformation of branch duct-type intraductal papillary mucinous neoplasms of the pancreas based on contrastenhanced endoscopic ultrasonography morphological changes: focus on malignant transformation of intraductal papillary mucinous neoplasm itself. Pancreas 2012;41:855-862.

23. Kamitani K, Kamitani T, Ono M, Toyoshima S, Mitsuyama S. Ultrasonographic findings of invasive micropapillary carcinoma of the breast: correlation between internal echogenicity and histological findings. Breast Cancer 2012;19:349-352.

24. Quaia E. Microbubble ultrasound contrast agents: an update. Eur Radiol 2007;17:1995-2008.

25. D'Onofrio M, Zamboni GA, Malago R, Mantovani W, Principe F, Gallotti $A$, et al. Resectable pancreatic adenocarcinoma: is the enhancement pattern at contrast-enhanced ultrasonography a preoperative prognostic factor? Ultrasound Med Biol 2009;35:19291937.

26. Nishida M, Koito K, Hirokawa N, Hori M, Satoh T, Hareyama M. Does contrast-enhanced ultrasound reveal tumor angiogenesis in pancreatic ductal carcinoma? A prospective study. Ultrasound Med Biol 2009;35:175-185. 